\title{
Biological polarized light reflectors in stomatopod crustaceans
}

Tsyr-Huei Chiou, Thomas W. Cronin, Roy L. Caldwell, Justin Marshall

Tsyr-Huei Chiou, Thomas W. Cronin, Roy L. Caldwell, Justin Marshall, "Biological polarized light reflectors in stomatopod crustaceans," Proc. SPIE 5888, Polarization Science and Remote Sensing II, 58881B (10 September 2005); doi: 10.1117/12.613117

SPIE. Event: Optics and Photonics 2005, 2005, San Diego, California, United States 


\title{
Biological polarized light reflectors in stomatopod crustaceans
}

\author{
Tsyr - Huei Chiou ${ }^{* a}$, Thomas W. Cronin ${ }^{a}$, Roy L. Caldwell ${ }^{\mathrm{b}}$, Justin Marshall ${ }^{\mathrm{c}}$ \\ ${ }^{a}$ Department of Biological Sciences, University of Maryland Baltimore County, Baltimore, MD, \\ USA 21250 \\ ${ }^{\mathrm{b}}$ Department of Integrative Biology, University of California, Berkeley, CA, USA 94720 \\ ${ }^{c}$ Vision, Touch and Hearing Research Centre, University of Queensland, Brisbane, Queensland \\ 4072, Australia
}

\begin{abstract}
Body parts that can reflect highly polarized light have been found in several species of stomatopod crustaceans (mantis shrimps). These polarized light reflectors can be grossly divided into two major types. The first type, usually red or pink in color to the human visual system, is located within an animal's cuticle. Reflectors of the second type, showing iridescent blue, are located beneath the exoskeleton and thus are unaffected by the molt cycle. We used reflection spectropolarimetry and transmission electron microscopy (TEM) to study the reflective properties and the structures that reflect highly polarized light in stomatopods. For the first type of reflector, the degree of polarization usually changes dramatically, from less than $20 \%$ to over $70 \%$, with a change in viewing angle. TEM examination indicates that the polarization reflection is generated by multilayer thin-film interference. The second type of reflector, the blue colored ones, reflects highly polarized light to all viewing angles. However, these reflectors show a slight chromatic change with different viewing angles. TEM sections have revealed that streams of oval-shaped vesicles might be responsible for the production of the polarized light reflection. In all the reflectors we have examined so far, the reflected light is always maximally polarized at around $500 \mathrm{~nm}$, which is close to the wavelength best transmitted by sea water. This suggests that the polarized light reflectors found in stomatopods are well adapted to the underwater environment. We also found that most reflectors produce polarized light with a horizontal $e$-vector. How these polarized light reflectors are used in stomatopod signaling remains unknown.
\end{abstract}

Keywords: biological signal, polarization vision, polarized light, stomatopod, structural polarization

\section{INTRODUCTION}

Visual signaling plays an important role in communication for animals that have eyes. Color or spectral based visual signals are commonly used by many terrestrial as well as aquatic animals in a variety of behavioral contexts. However, color signals have several drawbacks, especially in aquatic environments where the photic environment is constantly changing due to absorption, reflection, and scattering of light by water, solutes, and suspended particles. To compensate for the attenuation and to ensure fidelity of signals, animals expressing spectral based signaling systems might use strategies such as changing coloration or tuning photoreceptors to achieve color constancy in different illumination conditions ${ }^{1}$.

\subsection{The benefit of using polarized light signal}

Some animal groups, especially nocturnal animals or those who live in the deep sea environment, get around the problem just described by using bioluminescence ${ }^{2}$. Although bioluminescent signals are immune to variations in illumination conditions especially in dim-light environment, this kind of active signaling system might reveal the position of the animal, thus incurring unwanted predation. While bioluminescence might be the only solution for animals who want to use visual signals in dark environment, passive signals are used by the majority of animals that live in brighter environments. One way to produce a reliable visual signal while keeping the risk of exposing oneself minimized is to use polarized light. While animals that have the ability to perceive polarization of light are not uncommon, polarized signals are still obscure to most other animals that are unable to see polarized light. Consequently polarization based

\footnotetext{
"chiou1@umbc.edu; phone 1410 455-2975; fax 1410 455-3875
} 
signals could serve as a concealed visual communication channel for certain animal groups ${ }^{3,4}$. Polarization vision (i.e. the ability to discriminate between different $e$-vector angles) has been developed in a variety of animal groups for use in navigation, contrast enhancement, and communication ${ }^{5}$. To use polarized light in navigation or contrast enhancement, all an animal needs is polarization vision. However, to constitute a polarized light signaling system, the animals not only have to employ polarization vision but also need a way to produce reliable polarized light signals.

\subsection{Biological polarized light reflectors}

Except for bioluminescence, most animals generate visual signals through differential reflection, absorption, or scattering of ambient light. To generate color signals, the reflectors could absorb, reflect, or scatter different wavelengths of light differently. Similarly, polarization reflectors could absorb, reflect, or scatter various proportions of light depending on its $e$-vector angles. The major ways to produce polarized light include the use of intrinsically dichroic molecules, reflection, scattering, or birefringence. Among these, polarized light produced by intrinsic dichroism depends on the arrangement of atoms within the molecule which cannot be altered by changing the shape or size of the material. The best known dichroic molecules found in the animal kingdom are visual pigments. The dichroism of visual pigments is the basis for polarization vision ${ }^{6}$. However, perhaps due to the difficulty of extracting and re-crystallizing specific molecules in biological systems, no biological polarized-light reflectors based on intrinsically dichroic molecules have been found to date.

The production of polarized light through reflection and scattering is dependent on physical properties such as the dimensions of particles, thicknesses of layers, or refractive indices of the materials as well as the refractive indices of the surrounding media. Polarized light produced simply by reflection or scattering is either partially or fully linearly polarized. The polarized light reflections produced by these types of reflectors usually also show an interference color. Structures of many animals, such as polarization reflecting iridophores in cephalopod molluscs ${ }^{7,8}$ and the scales of both fishes ${ }^{9-11}$ and butterflies ${ }^{12-15}$, are known to produce highly linearly polarized light reflections by multilayer reflection and possibly scattering as well. Besides butterflies, the cuticles of several other arthropods such as beetles and crustaceans are also known to have reflectors based on multilayered structures ${ }^{16-18}$. A major component of arthropod cuticles, chitin crystallites, is a molecule with intrinsic birefringence. Furthermore, incorporation of other molecules into the cuticle has been found to be able to increase the birefringence of certain cuticular structures. A good example of this is the iridescent exoskeleton of scarab beetles, in which $70 \%$ of the cuticle volume is made up of uric acid ${ }^{19}$. Due to the birefringence, properly organized cuticle may produce either linearly or circularly polarized light, depending on both the molecular and physical properties of the materials.

The purpose of this study was to investigate the polarization reflections produced by stomatopod crustaceans. It became clear during our investigation that the polarization reflectors in stomatopod crustaceans are of at least two types. Examples of both reflector types, spectral properties of the reflected light, and the morphology of the structures responsible for the production of polarization reflections in both reflector types are described in this report.

\section{MATERIAL AND METHODS}

\subsection{Animals and general procedures}

Stomatopods, commonly known as mantis shrimps, were collected from near Key Largo, Florida, USA, Catalina Island, California, USA, or from Lizard Island, Queensland, Australia. Species used for this study include Haptosquilla trispinosa, Haptosquilla glyptocercus, Hemisquilla californiensis, Odontodactylus scyllarus, and Odontodactylus havanensis. Animals, except for $H$. californiensis which we did not house in the laboratory, were individually maintained in marine aquaria filled with artificial seawater (Instant Ocean, specific gravity 1.020 to 1.023 at $24^{\circ} \mathrm{C}$, Aquarium Systems Inc.). Each aquarium was illuminated under a LD 12:12 light cycle with a timer-controlled 15W fluorescent lamp (Power-Glo $18000 \mathrm{~K}$, Rolf C. Hagen Corp.), and the temperature was controlled at $25 \sim 27^{\circ} \mathrm{C}$. The animals were regularly fed with either fresh brine shrimp or frozen shrimp. Experimental animals were euthanized with icy cold sea water shortly after removal from the aquarium. Varying the temperature between $25 \sim 27^{\circ} \mathrm{C}$ and $0^{\circ} \mathrm{C}$ did not generate any noticeable color or polarization property changes on the animals. With the help of a piece of polarization filter held under a dissecting microscope, the polarization-reflecting body parts were identified and subsequently ablated for the 
following experiments. Images under the light microscope were taken with an Olympus C5050 Zoom digital camera which was adapted to the microscope with a universal digital camera microscope coupler (Edmund Optics).

\subsection{Measurements and analysis of reflectance spectra}

For measuring reflection properties, materials removed from the animal were submersed in sea water at room temperature throughout the course of the measurements. The spectral properties of the reflected light were examined with a spectrometer (USB2000, Ocean Optics Inc.) through an optic fiber. Depending on the area of interest, the optic fiber was attached to either a compound microscope $\left(\leq 1 \mathrm{~mm}^{2}\right)$ or an optic fiber condenser $\left(>1 \mathrm{~mm}^{2}\right)$. In either case, a polarization filter (HNP'B) was fitted in front of the optics with a custom-made rotatable adaptor. Three sets of spectral data plus a measurement of dark noise were collected for each sample, with the polarization filter positioned at $0^{\circ}, 45^{\circ}$, and $90^{\circ}$ with $0^{\circ}$ pointing horizontal to the right of the observer. They were referenced to another set of spectra recorded in the same conditions from a Teflon tape used as a diffuse white standard. From these measurements we calculated the spectral properties of reflectance from the sample as well as the $e$-vector angle and the partial polarization at $1 \mathrm{~nm}$ intervals from 350 to $850 \mathrm{~nm}$. The above calculations were performed by a custom-written program based on the equations derived by Wolff and Andreou 20 .

\subsection{Preparations for structure examination}

Specimens were fixed with $2.5 \%$ glutaraldehyde in PEMS buffer $\left(0.1 \mathrm{M}\right.$ PIPES, $0.01 \mathrm{M}$ EGTA, $0.0005 \mathrm{M} \mathrm{MgCl}_{2}$, $0.15 \mathrm{~g} / \mathrm{ml}$ sucrose, $\mathrm{pH}=7.1$ ) overnight at $4^{\circ} \mathrm{C}$ and post fixed with $1 \%$ osmium tetroxide in PEMS for 2 hours on ice. If required, the specimens were decalcified with several changes of $1 \%$ EGTA in $0.1 \mathrm{M}$ cacodylate buffer $(\mathrm{pH}=7.0)$ at $4^{\circ} \mathrm{C}$ for 2 weeks prior to dehydration. During dehydration, specimens were stained en bloc with $2 \%$ uranyl acetate in absolute ethanol for 2 hours at room temperature. Following dehydration, the specimens were infiltrated with gradient of 1:1 (w/w) Epon:Spurr's resins in acetone at room temperature and cured in 100\% of the same resin for 24 hours at $70^{\circ} \mathrm{C}$ under a slight vacuum. Thin sections of $60 \sim 90 \mathrm{~nm}$ thickness from the specimen blocks were obtained and examined without further staining procedure. Examination and photographs of the thin sections were carried out on a Zeiss transmission electron microscope (EM-10CA) with an accelerating voltage of $60 \sim 80 \mathrm{kV}$.

\section{RESULTS}

\subsection{Types of polarization reflectors}

The polarization reflectors found in stomatopods can be conveniently categorized into two types. The first type of reflector looks pink or red to the human visual system, and henceforth will be called the "red polarization reflector". The red polarization reflectors usually cover relatively large areas and are found mainly in calcified cuticle in various structures such as the antennal scales of $H$. californiensis and $O$. scyllarus, the uropods of $O$. havanensis and $O$. scyllarus, and the carapace of H. californiensis (Fig. 1A-D). Sexual dimorphism was found in the uropods of $O$. scyllarus, in that polarization reflections were only found in sexually mature males. In terms of coverage, the smallest red polarization reflector was found in $H$. glyptocercus, located on the dorsal side of the intersegmental membrane (i.s.m.) between the last two abdominal segments (Fig. 1E). However, altering the tension of the i.s.m. in the Haptosquilla species did not generate any observable polarization reflection changes, indicating that the polarization reflection was either generated by structures outside the i.s.m. or by a rigid structure within the i.s.m.. Since the i.s.m. needs to be highly flexible for proper function, it is most likely that the red polarization reflectors found in Haptosquilla species are also located in calcified cuticle near the i.s.m.. Consequently, all the red polarization reflectors mentioned above were found in the molt (or ecdyses) but not in the newly molted animal (e.g. Fig. 1D, E). Interestingly, during the processes of electron microscopical preparation, we also found that the optic properties of the red polarization reflectors were not affected by prolonged exposure to aldehyde fixatives ( $>1$ year) or decalcification media ( $>2$ weeks). Although we did not systematically test the effect of surrounding media on the optic properties of the polarization reflectors, short term $(<1$ month) submersion in either distilled water or glycerol did not seem to affect their optical properties in any obvious way. 
The second type of reflector is called the "blue polarization reflector" due to its iridescent blue appearance to human eyes. Compared to typical red reflectors, the blue polarization reflectors were smaller in size, and unlike the red types, were located underneath the cuticle. As a result, the optical properties of the blue polarization reflectors remained unchanged throughout the molt cycle. Blue polarization reflectors were found most commonly in the first maxillipeds of stomatopods (Fig. 2A). Species that have polarization-reflecting maxillipeds include H. californiensis, Haptosquila trispinosa, Haptosquilla banggai, Haptosquilla nefanda, H. pulchella, and Odontodactylus latirostris. The largest blue polarization reflector we have seen is one that covers the entire antennae of Hemisquilla californiensis (Fig. 2B). In all cases, the blue polarization reflectors are composed of layers of parallel fibers which are visible under the dissecting microscope (left frame of Fig. 2A). While all the fibers in one reflector are parallel to each other, each fiber is also parallel to the long axis of the appendage within which it resides. These fibers form a carpet-like structure which blankets the rest of the tissues underneath the cuticle. Although the blue reflectors survive throughout molting cycle, to be optically effective, the cuticle above it must be transparent.
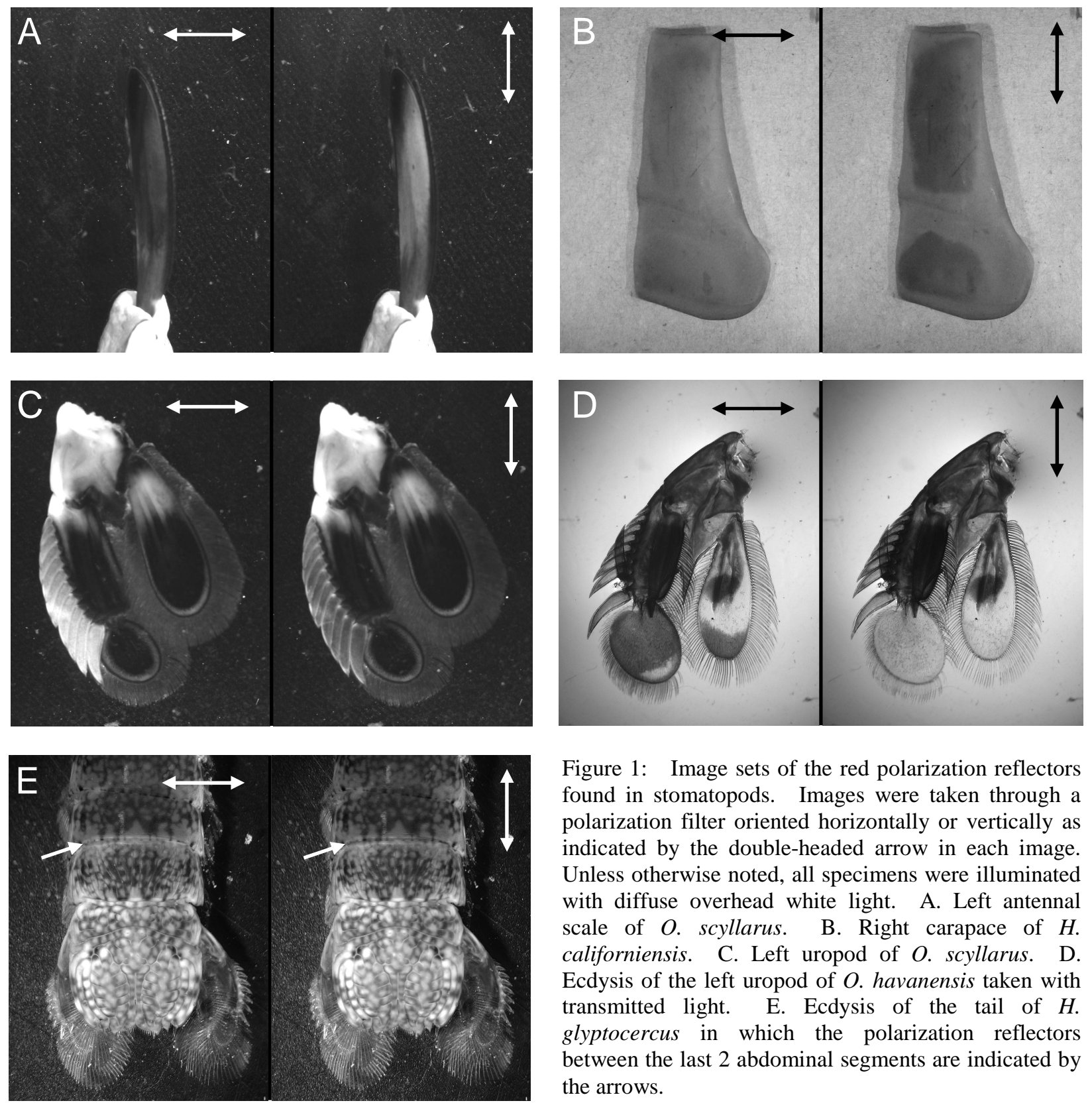

Figure 1: Image sets of the red polarization reflectors found in stomatopods. Images were taken through a polarization filter oriented horizontally or vertically as indicated by the double-headed arrow in each image. Unless otherwise noted, all specimens were illuminated with diffuse overhead white light. A. Left antennal scale of $O$. scyllarus. B. Right carapace of $H$. californiensis. C. Left uropod of $O$. scyllarus. D. Ecdysis of the left uropod of $O$. havanensis taken with transmitted light. E. Ecdysis of the tail of $H$. glyptocercus in which the polarization reflectors between the last 2 abdominal segments are indicated by the arrows. 

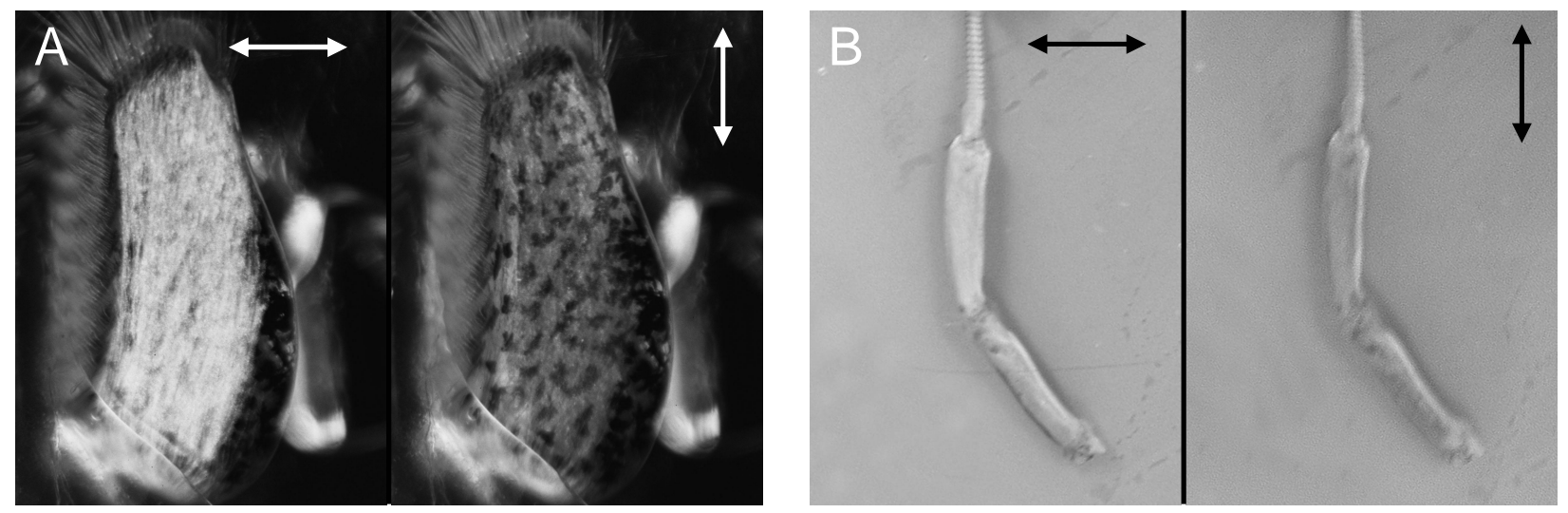

Figure 2: Image sets of the blue polarization reflectors from the right first maxilliped of $H$. trispinosa (A) and the right antenna of $H$. californiensis (B). As in Fig. 1, the images were taken through a polarization filter oriented horizontally or vertically as indicated by the double-headed arrow in each image. Both image sets were taken under overhead white light illumination.

\subsection{Spectral properties of the polarization reflections}

Spectral properties within each type of polarization reflector were qualitatively similar to one another (Fig. 3A, B). The polarization spectra of the red polarization reflectors always have a fairly sharp peak at around $450 \mathrm{~nm}$ to $550 \mathrm{~nm}$. Beyond $\sim 600 \mathrm{~nm}$ the degree of polarization drops to practically 0\% in most red polarization reflectors (Fig. 3A). However, the red polarization reflectors in $H$. californiensis and $H$. glyptocercus still have $\sim 25 \%$ and $\sim 5 \%$ polarization respectively in the $600 \mathrm{~nm}$ to $800 \mathrm{~nm}$ spectral range (Fig. 3A). Since the red polarization reflectors in both $H$. californiensis and $H$. glyptocercus are located on a convex cuticle, this weak yet constant polarization in the long wavelength range could be the consequence of surface reflections. On the other hand, the polarization spectra of the blue polarization reflectors always have a very broad peak. The highest degree of polarization for most blue polarization reflectors reaches $\sim 65 \%$, peaking between 500 to $600 \mathrm{~nm}$ (Fig. 3B). From $600 \mathrm{~nm}$ to $800 \mathrm{~nm}$ the degree of polarization gradually decreased to below $10 \%$. The spectral curves usually have an inflection point at around $700 \mathrm{~nm}$ to $750 \mathrm{~nm}$. As a result, a second peak appears at $\sim 750 \mathrm{~nm}$ to $\sim 770 \mathrm{~nm}$ in the polarization spectra of some blue polarization reflectors (THICK curve in Fig. 3B and 3D).

The spectral polarization of light reflected from both red and blue polarization reflectors varied as viewing angle changed. When the direction of observation was parallel to surface normal of the specimen when illuminated with diffused white light from $45^{\circ}$, the antennal scale of $O$. scyllarus (a red polarization reflector) showed little or no polarization (Fig. 3C). However, under the same experimental conditions, the first maxilliped of $H$. trispinosa (a blue polarization reflector) exhibited over $50 \%$ polarization at $\sim 550 \mathrm{~nm}$ (Fig. 3D). We then gradually increased the angles between the direction of observation and the surface normal of the specimen by tilting it toward (and beyond) the direction of illumination. For the antennal scale of $O$. scyllarus, increasing the tilting angle from $0^{\circ}$ to $60^{\circ}$ enhanced the degree of polarization from below $10 \%$ to over $83 \%$ (Fig. 3C). As the degree of polarization increased, the peak of the polarization spectrum slightly shifted from $450 \mathrm{~nm}$ when viewing from the surface normal toward near $530 \mathrm{~nm}$ at a $60^{\circ}$ angle of tilt. When the tilt angle of a maxilliped of $H$. trispinosa was increased from $0^{\circ}$ to $15^{\circ}$, the degree of polarization also increased slightly. While the polarization became stronger, the peak of the polarization spectrum curve was shifted toward a shorter wavelength (Fig. 3D). Further increases of the tilt angle of the blue polarization reflector reversed the above changes and resulted in a lower degree of polarization which peaked at a longer wavelength (i.e. from $15^{\circ}$ to $60^{\circ}$ tilt angle the peak of the degree of polarization shifted from $62.6 \%$ at $510 \mathrm{~nm}$ to $20.3 \%$ at $640 \mathrm{~nm}$; Fig. 3D).

\subsection{Fine structures of polarization reflectors}

As we have previously indicated, all the red polarization reflectors found in stomatopods were located in calcified cuticle. Further examination of a hand sectioned cuticle under the dissecting microscope has revealed that, at least in 


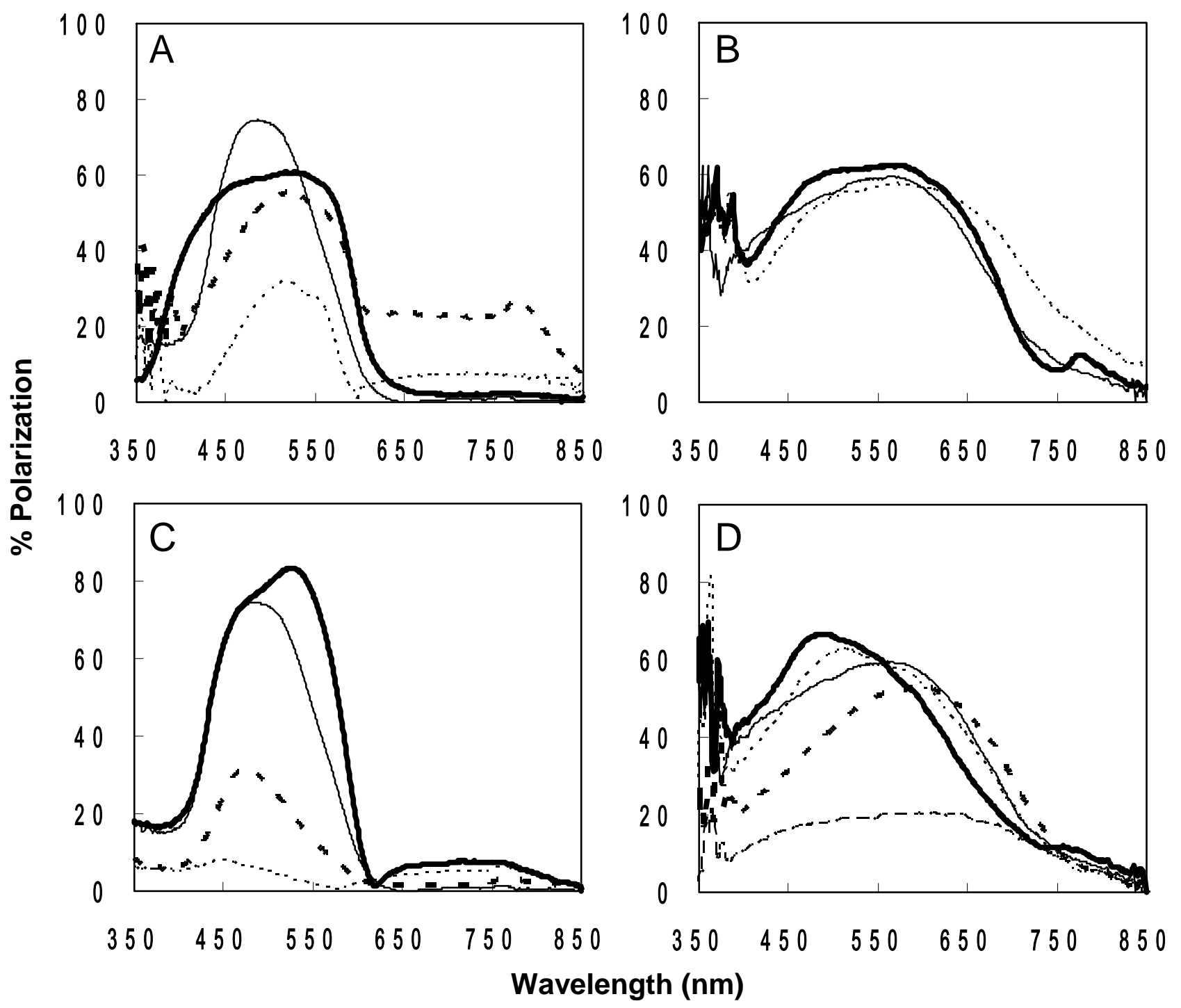

Figure 3: Spectral properties of the polarization. A. The polarization spectra of different red polarization reflectors. The uropod of $O$. scyllarus, THICK curve; the antennal scale of $O$. scyllarus, THIN curve; the carapace of $H$. californiensis, THICK DOTTED curve; the i.s.m. of $H$. glyptocercus, THIN DOTTED curve. B. The polarization spectra of different blue polarization reflectors. The antenna of $H$. californiensis, THICK curve; the maxilliped of $H$. californiensis, THIN curve; the maxilliped of $H$. trispinosa, THIN DOTTED curve. C. The polarization spectra of an antennal scale of $O$. scyllarus tilted at various angles. THIN DOTTED curve represents tilt angle of $0^{\circ}$; THICK DOTTED curve, $20^{\circ}$; THIN curve, $40^{\circ}$; THICK curve, $60^{\circ}$. D. The polarization spectra of a maxilliped of $H$. trispinosa tilted at various angles. THIN curve were obtained from specimen with $0^{\circ}$ tilt angle; THICK curve, $15^{\circ}$; THIN DOTTED curve, $30^{\circ}$; THICK DOTTED curve, $45^{\circ}$; THIN DASHED curve, $60^{\circ}$.

the antennal scale of $O$. scyllarus and the carapace of $H$. californiensis, only the exocuticle was optically active. Transmission electron microscopic images of the optically active part of the cuticle show that the polarization-reflecting cuticle of stomatopods contains a layered structure that is parallel to the surface (Fig. 4A). Each layer in the cuticle is composed of alternating laminar membranes. The lamellate membranes are parallel to each other but oblique to the cuticle's surface (Fig. 4A). The thickness of the lamellate membrane measured within one section might vary from $\sim 200 \mathrm{~nm}$ to $\sim 1000 \mathrm{~nm}$. Nevertheless, fibrous structures that are uniform in thickness and orientation within the less electron dense type of the lamellate membrane are visible in high magnification electron micrographs (Fig. 4B). 

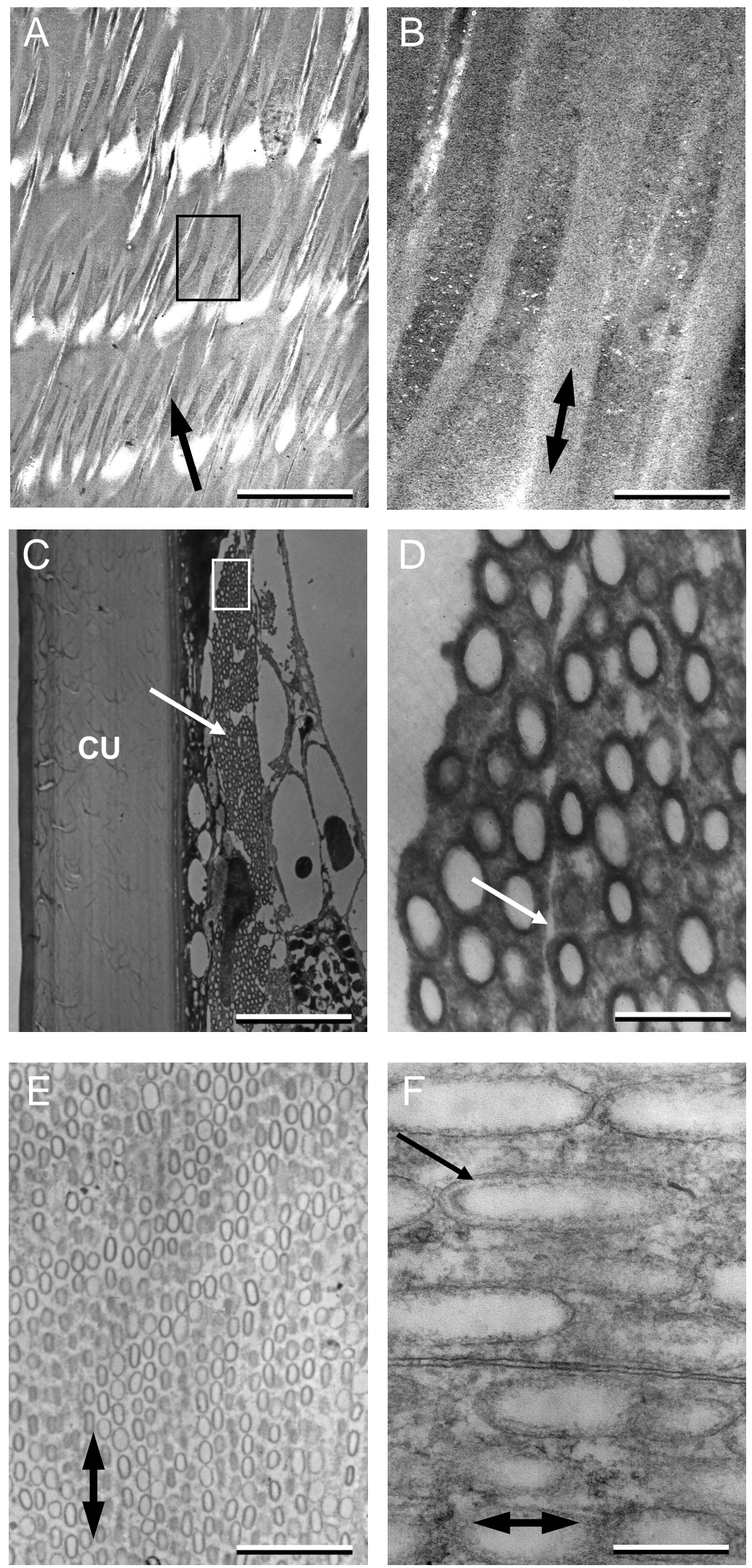

Figure 4: Transmission electron microscopic images of polarization reflectors. A. Cross section of the carapace of $H$. californiensis showing the lamellate structure in the cuticle. Arrow indicates the direction toward the external surface of the carapace. Scale bar, 6 $\mu \mathrm{m}$. B. High magnification micrograph of the marked area in A. The orientation of chitin microfibrils is indicated by a double-headed arrow. Scale bar, $1 \mu \mathrm{m}$. C. Cross section of first maxilliped of $H$. trispinosa showing the small vesicles (arrow) underneath the cuticle (CU). Scale bar, $5 \mu \mathrm{m}$. D. Enlargement of the marked area in C. The vesicles are grouped into streams with a very narrow gap (arrow). Scale bar, $0.45 \mu \mathrm{m}$. E. Same as C but sectioned parallel to the cuticle to show the alignment of the vesicles. The doubleheaded arrow indicates the long axis of the maxilliped. Scale bar, $3 \mu \mathrm{m}$. F. Sagittal section of the first maxilliped of $H$. californiensis. Each vesicle has two layers of cell membrane. The double-headed arrow indicates the long axis of the maxilliped. Scale bar, $0.24 \mu \mathrm{m}$. 
All the blue polarization reflectors have large numbers of vesicles underneath the cuticle (Fig. 4C). Groups of vesicles are enclosed by a membrane (Fig. 4D) and form thread-like structures which are visible under a dissecting microscope. As mentioned earlier, these threads are parallel to the long axis of the maxilliped or antenna (left panel of Fig. 2A). When sectioned parallel to the threads, it becomes clear that the vesicles not only have an elongated shape but also a consistent orientation (Fig. 4E, F). The parallel sections also show that the vesicles within each thread are compact and highly organized. In high-maginification cross sectional micrographs, these vesicles seemed to have different sizes (Fig. 4D). However, from the organization of these vesicles and micrographs of these vesicles from different section planes, we believe that all the vesicles are about the same size. All the vesicles looked like elongated olives with the long axis parallel to the threads they were within. Judging from the shape and the numbers of the vesicles in different sectional planes, we were able to determine that in the maxilliped of $H$. trispinosa, the vesicles were are approximately $700 \mathrm{~nm}$ in length and $300 \mathrm{~nm}$ in diameter, while in the maxilliped and antenna of $\mathrm{H}$. californiensis, the vesicles were $\sim 600 \mathrm{~nm}$ in length and $\sim 200 \mathrm{~nm}$ in diameter.

\section{SUMMARY AND CONCLUSIONS}

In this paper we have described the spectral properties and morphology of several polarized-light reflectors found in stomatopod crustaceans. From color, polarization spectra, and fine structure, these polarized-light reflectors can be sorted into two groups. We named these two groups "red" and "blue" by their color appearance to our visual system. However, the color spectra of the specimen are not coinside with that of polarization. Polarization spectra of both reflector types show a high degree of polarization at roughly $450 \mathrm{~nm}$ to $550 \mathrm{~nm}$. When the polarization reflections were measured from different angles, both reflector types show changes in polarization spectra, but the directions of their changes were different. Nevertheless, the spectral polarization result implies that these polarization reflectors are based on a photonic structure of some sort instead of on intrinsically dichroic molecules. From electron micrographs, we have shown that the red polarization reflectors are based on multilayered structures while the blue ones are based on scattering of light by oval shaped vesicles. However, the components of the layers or materials within the vesicles as well as their physical properties remain unknown.

\section{ACKNOWLEDGEMENTS}

We would like to thank the staff of the Lizard Island Research Station for support and assistance. Funding for this research was provided by the National Science Foundation under Grant Number IBN-0235820 and by the Air Force Office of Scientific Research under Grant Number 02NL253 and by the NOAA/NURC Florida Keys Program at Key Largo, Florida.

\section{REFERENCES}

1. Cheroske, A.G., T.W. Cronin, and R.L. Caldwell, "Adaptive color vision in Pullosquilla litoralis (Stomatopods, Lysiosquilloidea) associated with spectral and intensity changes in light environment", The Journal of Experimental Biology, 206, 373-379, 2003.

2. Herring, P.J., "Species abundance, sexual encounter and bioluminescent signalling in the deep-sea", Philosophical Transactions of the Royal Society of London. Series B, 355, 1273-1276, 2000.

3. Nilsson, D.E. and E.J. Warrant, "Visual Discrimination: Seeing the third quality of light", Current Biology, 9, R535R537, 1999.

4. Marshall, N.J., T.W. Cronin, N. Shashar, and M. Land, "Behavioural evidence for polarisation vision in stomatopods reveals a potential channel for communication", Current Biology, 9, 755-758, 1999.

5. Horvath, G. and D. Varju, Polarized light in animal vision: polarization patterns in nature, 448, Springer-Verlag, Berlin Heidelberg, 2003.

6. Rossel, S., "Polarization sensitivity in compound eyes", Facets of Vision, D.G. Stavenga and R.C. Hardie (eds). 298316, Springer-Verlag: Berlin, Heidelberg, 1989.

7. Mathger, L.M. and E.J. Denton, "Reflective properties of iridophores and fluorescent 'eyespots' in the Loliginid squid Alloteuthis subulata and Loligo vulgaris", The Journal of Experimental Biology, 204, 2103-2118, 2001.

8. Shashar, N., D.T. Borst, S.A. Ament, W.M. Saidel, R.M. Smolowitz, and R.T. Hanlon, "Polarization reflecting iridophores in the arms of the squid Loligo pealeii", Biological Bulletin, 201, 267-268, 2001. 
9. Denton, E.J., F.R.S., and J.A.C. Nicol, "Polarization of light reflected from the silvery exterior of the bleak, Alburnus alburnus", Journal of the Marine Biological Association of the United Kingdom, 45, 705-709, 1965.

10. Denton, E.J. and J.A.C. Nicol, "Reflexion of light by external surfaces of the herring, Clupea harengus", Journal of the Marine Biological Association of the United Kingdom, 45, 711-738, 1965.

11. Denton, E.J. and J.A.C. Nicol, "Studies on reflexion of light from silvery surfaces of fishes, with special reference to the bleak, Alburnus alburnus", Journal of the Marine Biological Association of the United Kingdom, 45, 683-703, 1965.

12. Vukusic, P., J.R. Sambles, and C.R. Lawrence, "Colour mixing in wing scales of a butterfly", Nature, 404, 457, 2000 .

13. Vukusic, P., J.R. Sambles, and H. Ghiradella, "Optical classification of microstructure in butterfly wing-scales", Photonic Science News, 6, 61-66, 2000.

14. Vukusic, P., J.R. Sambles, C.R. Lawrence, and R.J. Wootton, "Now you see it - now you don't", Nature, 410, 303, 2001.

15. Vukusic, P., J.R. Sambles, C.R. Lawrence, and R.J. Wootton, "Limited-view iridescence inthe butterfly Ancyluris meliboeus", Proceedings of the Royal Society of London. Series B, 269, 7-14, 2002.

16. Neville, A.C., "Physical properties", Biology of the arthropod cuticle, D.S. Farner, W.S. Hoar, J. Jacobs, H. Langer, and M. Lindauer (eds). 319-374, Springer-Verlag: Berlin, Heidelberg, 1975.

17. Neville, A.C. and B.M. Luke, "Form optical activity in crustacean cuticle", Journal of Insect Physiology, 17, 519526, 1971.

18. Neville, A.C. and S. Caveney, "Scarabid beetle exocuticle as an optical analogue of cholesteric liquid crystals", Biological Reviews, 44, 531-562, 1969.

19. Caveney, S., "Cuticle reflectivity and optical activity in scarab beetles: the role of uric acid", Proceedings of the Royal Society of London. Series B, 178, 205-225, 1971.

20. Wolff, L.B. and A.G. Andreou, "Polarization camera sensors", Image and Vision Computing, 13, 497-510, 1995. 